\title{
Approche cognitive de la régulation de la motivation
}

Christophe Gernigon

\section{Citer ce document / Cite this document :}

Gernigon Christophe. Approche cognitive de la régulation de la motivation. In: Les Cahiers de l'INSEP, n¹4-15, 1996. La récupération en sport : approches des techniques et des moyens. pp. 75-81;

doi : https://doi.org/10.3406/insep.1996.1167

https://www.persee.fr/doc/insep_1241-0691_1996_num_14_1_1167

Fichier pdf généré le 24/02/2020 


\section{APPROCHE COGNITIVE DE LA RÉGULATION DE LA MOTIVATION}

\section{Christophe GERNIGON}

Laboratoire de psychologie de l'INSEP

L'adaptation aux conditions extrêmes de la performance relève de processus sollicités immédiatement après une performance jusqu'à l'ultime moment précédant une nouvelle performance. Entre les deux, de nombreux facteurs agissant sur les représentations du sportif peuvent contribuer à altérer ou à optimiser l'état motivationnel de celui-ci. L'objectif de la récupération ne consiste alors plus seulement à recouvrer ses forces physiques et mentales, mais aussi à mieux gérer les mécanismes cognitifs qui président aux comportements d'abandon et de résignation ainsi qu'aux attitudes les plus persévérantes et combatives. Deux moments, particulièrement en prise avec les mécanismes de la motivation, sont probablement les plus saturés en facteurs déterminant la qualité de la récupération au sens où nous venons de l'envisager (l'analyse de la prestation sportive passée et la détermination des projets immédiats ou différés).

\section{L'analyse de la prestation passée}

L'épreuve sportive, en tant qu'instant de vérité, possède une valeur informationnelle, motivationnelle et émotionnelle. L'analyse des résultats, et de la manière dont ceux-ci sont obtenus, apparaît alors déterminante pour le maintien d'un haut niveau d'engagement du sportif. Le résultat apparaît seulement comme l'un des facteurs pouvant influencer les processus cognitifs impliqués dans les comportements d'abandon ou de persévérance. Ces processus, ou médiateurs cognitifs, sont des représentations relatives au contrôle de la situation par l'athlète et à sa propre compétence. 


\section{1 - Préserver le sentiment de contrôle des événements}

Le sportif n'échappe pas à une tendance générale qui conduit l'être humain à s'interroger sur le pourquoi de ses résultats. Toutefois, l'impact émotionnel consécutif à une prestation sportive dégrade l'objectivité de I'analyse causale. Aussi convient-il d'éviter toute analyse technique ou tactique à chaud d'une compétition sportive. De l'analyse causale des résultats dépendent des représentations sur la contrôlabilité de l'environnement et des événements. Ces représentations sont à l'origine de conséquences déterminantes pour le sportif. Ainsi, la perception d'indépendance entre les comportements et les résultats obtenus est génératrice de résignation. Les déficits qui en découlent affectent directement la performance, la motivation ainsi que la sphère émotionnelle. Les caractéristiques des causes perçues comme étant responsables des résultats obtenus jouent sur la gravité des déficits ainsi observés. Si la mise en avant de causes internes (facteurs personnels) et stables (ces facteurs interviennent régulièrement), après un succès, s'avère propice à l'adoption de comportements persévérants, l'attribution d'un échec à ces mêmes causes devient préjudiciable. En revanche, une attribution à des causes internes, instables, invoquant la quantité d'effort déployée par exemple, préserve la perception de contrôle sur les événements, ceci quel que soit le résultat. Ainsi, l'aspect circonstanciel d'un échec qui sera perçu comme surmontable, pour peu que l'on décide d'investir davantage d'effort, n'affectera pas la motivation du sujet.

Dévoiler les causes objectives d'un résultat implique, dans tous les cas, de souligner le caractère réversible de celles-ci. Les facteurs considérés comme responsables d'un succès devront être perçus comme labiles en cas de réduction de l'intensité du travail à l'entraînement. II en va de même pour les raisons d'une contre-performance qui devront apparaître comme éphémères si le sportif consent à investir suffisamment d'effort. Les gratifications, sous formes de récompenses diverses, constituent des renforçateurs externes de la motivation. Ces incitations surajoutées aux motifs intrinsèques de la pratique sportive sont susceptibles d'exercer un pouvoir contrôlant sur le sportif. Si elles ne semblent pas affecter l'activité apparente du sujet, à long terme un effet négatif s'observe fré- 


\section{LA RÉCUPÉRATION EN SPORT}

quemment sous la forme d'une réduction de l'activité, voire d'un abandon. Bien qu'elle apparaisse inévitable dans le contexte sportif, il importe de limiter la prépondérance du contrôle externe exercé par les différentes gratifications.

\section{2 - Préserver l'estime de soi}

Les comportements que les personnes manifestent lorsqu'elles s'appliquent à bien réaliser une tâche, à rechercher des normes d'exigence, d'excellence ou de performance, à se fixer des buts élevés (comportements d'accomplissement), sont mus par un processus majeur qu'il convient d'entretenir : le sentiment de compétence. Les représentations que l'on se forge de sa propre compétence participent à la construction de l'estime de soi. Le problème réside alors dans la difficulté qu'il y a à préserver ce sentiment de compétence et l'estime de soi après une défaite ou une contre-performance. En fait, le résultat n'aurait pas de valeur incitatrice en lui-même, si ce n'est dans l'information de compétence qu'il fournit au sujet. La construction d'un tel sentiment ne peut se faire à partir de succès trop aisés à obtenir. C'est pourquoi la tâche doit présenter pour le sujet un degré optimal de défi. En outre, il importe de ne jamais dévaloriser la tâche ou l'adversaire, ceci quel que soit le résultat. Le contraire présenterait le risque d'affecter gravement l'estime de soi par la dégradation du sentiment de compétence chez l'athlète.

Il existe principalement deux manières de se sentir compétent. La première répond à une recherche de maîtrise des tâches à réaliser. Elle s'inscrit dans une logique de comparaison temporelle auto-référée, c'està-dire que la compétence est perçue à partir de ses propres progrès. La seconde repose sur la comparaison sociale, elle est donc normativement référée. Selon la manière adoptée pour se représenter leur compétence, les individus auront tendance à se fixer des buts investis sur la maîtrise des tâches dans le cas d'une représentation auto-référée, ou bien des buts de promotion de l'ego dans le cas d'une représentation normativement référée.

Le type de but développé, combiné avec le niveau d'habileté perçue, 
entraîne d'importantes conséquences motivationnelles. Une personne s'estimant habile ou compétente dans un domaine particulier manifestera des comportements de persévérance, ceci quel que soit le type de but développé (investi sur la tâche ou sur l'ego). En revanche, si elle s'estime incompétente, cette personne s'attachera à déployer des efforts seulement si elle poursuit des buts investis sur la tâche. Si, par contre, ses buts motivationnels sont exclusivement orientés vers l'ego, elle mettra en oeuvre des stratégies visant à échapper aux jugements défavorables des autres (comme s'engager dans des compétitions de moindre niveau, abandonner, etc.). Le rôle des orientations d'accomplissement des sportifs apparaît donc déterminant dans l'adoption des comportements d'assiduité ou d'abandon. Ces orientations subissent l'influence de la compétition, laquelle contribue à développer chez les individus des buts orientés vers l'ego. Par la comparaison sociale qu'elle instaure, elle constitue une arme à double tranchant jouant le rôle d'un outil "motivationnel " au service des seuls gagnants. Aussi est-il important également de se préoccuper d'entretenir les buts de maîtrise parallèlement aux buts d'implication de l'ego développés de manière inévitable par la compétition.

Le contexte de pratique, ou climat, influence les orientations " motivationnelles". Les éléments constitutifs de la structure de ce contexte, responsables du modelage des orientations " motivationnelles" sont le type de tâches, la nature de l'autorité, les récompenses, l'organisation des groupes et les modalités d'évaluation. Certaines de ces caractéristiques du climat " motivationnel " sont impliquées dans l'analyse de la prestation passée. Ainsi, les gratifications ne doivent pas être décernées au seul vu des résultats, mais doivent également souligner les progrès collectifs et personnels constatés. Ceci plaide bien sûr en faveur d'une évaluation du sportif à partir de critères individuels prenant en compte ses propres progrès. Le souci de préserver le sentiment de compétence entre donc dans une logique de récupération mentale et nécessite une organisation temporelle du "debriefing " qui fait suite à une compétition. Il est préférable d'aborder en premier lieu les points positifs tant d'ordre technique et tactique qu'énergétique, mental, émotionnel et relationnel, même si ceux-ci sont peu nombreux, notamment en cas de 


\section{LA RÉCUPERATION EN SPORT}

contre-performance. Dans un second temps, celui de l'analyse causale des aspects négatifs, il s'agira davantage de mettre en évidence les facteurs circonstanciels de ses propres faiblesses et des points forts de l'adversaire avec le maximum d'objectivité et de sérénité. Les réprimandes publiques demeurent généralement inefficaces, surtout si elles s'adressent à un ou à quelques individus. Enfin, une perspective positive subordonnée aux efforts à fournir sera évoquée en insistant sur le fait que les carences constatées peuvent être comblées lors des prochains entraînements.

\section{La détermination des projets}

Chez l'athlète, les objectifs sont essentiellement déterminés en regard d'échéances choisies dans le panel de compétitions qui jalonnent la saison sportive, la carrière. Les conséquences qui se dégagent des études concernant la fixation de buts (goal setting) permettent de guider les choix du sportif et de son entraîneur vers des objectifs plus pertinents au plan "motivationnel ". Par ailleurs, la manière dont les projets sont déterminés influence également l'engagement d'une personne.

\section{1 - Fixer des buts pertinents}

Deux facteurs essentiels déterminent le caractère attractif des objectifs à atteindre par le sportif : la valeur que représente la réalisation du but pour l'athlète et l'espérance de succès que celui-ci nouait à l'égard de ce but. Les objectifs doivent représenter un intérêt pour le sportif. Le besoin de manifester sa compétence l'amène à rechercher des enjeux de plus en plus élevés, mais aussi à l'issue de plus en plus incertaine. Si la difficulté représente une source de motivation, le défi à relever ne peut être démesurément élevé. Il s'agit bien d'un défi optimal pour les ressources dont dispose un athlète à un moment donné de la saison sportive ou de sa carrière. La faisabilité du projet demeure indispensable à la prévention du découragement. Pour les mêmes raisons, l'accessibilité potentielle des buts répond à des critères de temporalité. L'atteinte de ces buts doit pouvoir s'envisager à court ou moyen terme. Cependant, la 
carrière d'un sportif est aussi ponctuée de grands rendez-vous éloignés de plusieurs mois, voire de quelques années (Jeux Olympiques, Championnats du monde dans certaines disciplines). De telles conditions de difficulté et d'éloignement temporel semblent peu propices au maintien de la motivation. II devient alors indispensable de subdiviser les buts terminaux en sous-buts hiérarchisés et programmés dans la difficulté et dans le temps (buts de niveau intermédiaire et proximaux). L'atteinte de ces sous-buts par le sportif constituera autant de points de repère sur sa progression personnelle, ceci l'incitant de surcroît à élaborer une représentation de sa compétence centrée sur la comparaison temporelle. Le choix des objectifs en sport réclame donc un équilibre entre le pouvoir attractif que représente l'enjeu de la réussite d'une performance extrêmement élevée et le pouvoir répulsif de la trop faible probabilité subjective de réaliser celle-ci en raison de sa difficulté relative aux possibilités du sportif ou de son éloignement temporel.

\section{2 - Favoriser l'autodétermination}

La complexité et le caractère personnel des paramètres à prendre en compte pour déterminer les objectifs les plus pertinents pour un sportif ne plaide pas en faveur d'un style de management autoritaire de l'entraîneur. De plus, le sentiment de contrôle interne de l'avenir proche ou plus éloigné représente un facteur essentiellement responsable des attitudes de persévérance (cf. supra). Certes, la fonction de guide de l'entraîneur n'est pas à remettre en cause tant l'athlète ne peut s'affranchir du recours à des compétences externes moins affectées par l'impact émotionnel des situations sportives. Mais un partage des pouvoirs est générateur d'une perception de contrôle sur les décisions pour le sportif. La relation est donc à considérer en terme de collaboration. Abandonner une partie du contrôle des décisions au sportif implique de la part de l'entraîneur l'acceptation d'une réduction de son propre pouvoir. Cette concession doit s'opérer sans préjudice quant à la pertinence des choix qui seront effectués. Ce partage du pouvoir engendre alors inévitablement un effort de partage des compétences, et donc une démarche d'information et de divulgation des connaissances de la part de l'entraîneur. 


\section{LA RÉCUPÉRATION EN SPORT}

Par ailleurs, l'élan démocratique serait vain si le sportif ne s'investissait pas de son côté dans un effort de formation personnelle. L'accroissement de ses capacités à prendre des responsabilités passe par un accès aux connaissances habituellement réservées au manager ainsi que par l'appropriation de la maîtrises de ses outils. La nécessaire personnalisation de la gestion d'une carrière sportive réclame donc un style de management démocratique qui se révèle être le plus propice aux attitudes persévérantes.

\section{Conclusion}

La récupération psychologique peut donc s'envisager selon une double fonctionnalité organisée sur une dimension a posteriori représentée par sa fonction réparatrice et sur une dimension a priori de nature anticipatrice ou préparatrice. Celles-ci sollicitent des processus fondamentalement impliqués dans les comportements de persévérance et de recherche d'excellence tels qu'ils peuvent être souhaités après ou avant une épreuve sportive. Les sentiments de contrôle et de compétence, ainsi que la manière dont ils peuvent se décliner, constituent les facteurs principalement responsables des comportements adaptatifs. La genèse et l'entretien de ces facteurs dépendent essentiellement de l'objectivité et du caractère démocratique d'un climat de maîtrise au sein duquel la performance sportive s'analyse et se prépare. 